\title{
El problema del abandono de las pretensiones imprescriptibles \\ The abandonment issue of the imprescriptible pretensions
}

\author{
LUIS ALFARO VALVERDE \\ Universidad Nacional Mayor de San Marcos
}

Resumen: En el presente artículo se analiza el problema del abandono en los procesos judiciales relacionados con las pretensiones imprescriptibles, con una especial mención de aquellas relativas al derecho de propiedad. Se critica la cuestión analizada en el Pleno Jurisdiccional Nacional Civil y Procesal Civil de 2016 sobre el particular. Se pone de manifiesto que la verdadera cuestión a resolverse es la referida a las pretensiones imprescriptibles como supuestos de improcedencia de abandono. Se expone razones que ponen en entredicho la disposición normativa que vincula el abandono procesal con dichas pretensiones. Justamente por la falta de argumentos fuertes que justifiquen tal relación, se propone (de lege ferenda) su necesaria enmienda legislativa.

Palabras clave: abandono procesal, prescripción, derecho de propiedad, pleno jurisdiccional nacional

\begin{abstract}
In this article, the issue around abandonment in judicial proceedings (Procedural abandonment) related with the imprescriptible pretensions will be analyzed, with a special mention to those relative to the right to property. The issue discussed in the National Jurisdictional Plenary of Civil Law and Civil Procedure of 2016 will be criticized. The true issue to be solved is revealed, it's the one related to the imprescriptible pretensions as case of inappropriateness of abandonment. It raises reasons that put in question the normative provision that links the procedural abandonment with those pretensions. Precisely because of the lack of strong arguments to justify the relation, a necessary legislative amendment is proposed.
\end{abstract}

Keywords: procedural abandonment, prescription, right of property, National Jurisdictional Plenary

CONTENIDO: I. INTRODUCCIÓN.- II. SENTIDO Y JUSTIFICACIÓN DEL ABANDONO.- III. EL ABANDONO EN UN MUNDO «DE OFICIO».- IV. EL ABANDONO ANALIZADO EN EL PLENO JURISDICCIONAL NACIONAL CIVIL.- V. EL PROBLEMA DE LAS PRETENSIONES IMPRESCRIPTIBLES.VII. REFLEXIONES FINALES.

\footnotetext{
* Universidad Nacional Mayor de San Marcos, Maestría con mención en Derecho Procesal; Universidad Nacional Mayor de San Marcos y Universidad Inca Garcilaso de la Vega. Doctorando en el Programa de Doctorat en Dret, Economia i Empresa de la Universidad de Girona. Máster en Derecho Público, especialidad Derecho Procesal por la Universidad Complutense de Madrid. Miembro e Investigador externo del Instituto Vasco de Derecho Procesal.

Código ORCID: 0000-0001-8433-4099. Correo electrónico: alfavalu@ hotmail.com
} 


\section{INTRODUCCIÓN}

Desde hace un tiempo - aunque no mucho- estamos siendo testigos del establecimiento y desarrollo de una institución de Common Law en nuestro sistema jurídico, nos referimos a la recepción jurídica del precedente (sobre la recepción del precedente en los sistemas de Civil Law, véase Taruffo, 2012). Este hecho viene generando múltiples secuelas en los «formantes» jurídicos ${ }^{1}$, al menos en la producción legislativa, en las diversas posturas teóricas y, sobre todo, en los pronunciamientos jurisdiccionales. Como consecuencia de ello, se puede observar algunas peculiares posiciones que van desde una aceptación irreflexiva, pasando por una posición escéptica, hasta propuestas moderadas que admiten su recepción, pero que abogan por su adaptación. Sin embargo, debemos reconocer que -a diferencia de sistemas de Civil Law de Europa continental ${ }^{2}$ - hemos sido extremadamente solícitos y acríticos en su asentimiento, especialmente normativo. Esto sucede también en algunos otros países de la región.

A la par de esta situación —quizá como una forma de resistencia encubierta de los jueces frente a dicha figura-, al interior del Poder Judicial se viene presentando otro fenómeno muy particular que, aunque se parece, no podría catalogarse propiamente como precedente ni menos como jurisprudencia (para una clara explicación sobre las diferencias entre precedente y jurisprudencia, véase Taruffo, 2007). Nos referimos a aquello que se ha convenido en llamar plenos jurisdiccionales ${ }^{3}$, los cuales son definidos como las «reuniones de Magistrados de la misma especialidad, de una, algunas o todas las Cortes Superiores de Justicia del país, orientadas a analizar situaciones problemáticas relacionadas al ejercicio de la función jurisdiccional; con la finalidad que mediante su debate y posteriores conclusiones se determine el criterio más apropiado

1 La teoría de los formantes y su producto, los formantes jurídicos (legal formants), fue elaborada en Italia por Rodolfo Sacco a partir de la lingüística estructuralista. La expresión «formante» procede de la fonética acústica, el estudio de la consistencia física de los sonidos vocales y de su difusión a través de un medio como, por ejemplo, el aire. En este ámbito, el formante indica la frecuencia de resonancia de los sonidos que tienen lugar en la cavidad oral y que caracterizan su timbre: permite descomponer esos sonidos y, sobre todo, poner de manifiesto e identificar sus distintos componentes (Somma, 2015, p. 154). En la comparación jurídica, el formante representa los componentes del derecho, entre los que se identifican, el formante legal - para dar cuenta de las reglas elaboradas por el legislador-, el formante doctrinal - contenido por preceptos elaborados por los teóricos - y el formante jurisprudencial - el cual coincide con las indicaciones emitidas por los tribunales- (Sacco, 1992, pp. 44ss).

2 Por las repercusiones que viene generando en nuestro país, la posición abierta y permisiva frente al precedente (el cual se origina-conviene recordar- en países del Common Law) no parece haber sido la actitud ideal ni la más apropiada. Tal vez, para hacer frente a los problemas de la falta de predictibilidad y uniformidad del derecho - entre otras «razones»-, deberíamos haber optado por trabajar y mejorar la institución de la jurisprudencia (propia de Civil Law), la cual, por su falta de comprensión y su casi nulo desarrollo normativo (dado que no tenemos reglas sobre su formación, requisitos, grado de su fuerza normativa, etcétera) ciertamente no cumplió a cabalidad su propósito. Empero, puesto que este no es el lugar para seguir con tal cuestión, dejo abierto el debate y el compromiso de analizarse en otro trabajo.

3 También en otros sistemas jurídicos, desde hace un tiempo, podemos encontrar figuras semejantes a la mencionada, así, en España existen los llamados acuerdos de pleno jurisdiccional. En este caso, se viene discutiendo el grado de vinculación que tendrían (Manjón-Cabeza, 2008, pp. 1-25). 
para cada caso concreto» (Centro de Investigaciones Judiciales, 2008). En otras palabras es la congregación de jueces, de diferentes grados y especialidades, para debatir algunas cuestiones problemáticas de la práctica jurisdiccional y asumir una posición por mayoría. La práctica estaría justificada normativamente en el artículo 116 de la Ley Orgánica del Poder Judicial:

Plenos jurisdiccionales

Artículo 116. .- Los integrantes de las Salas Especializadas, pueden reunirse en plenos jurisdiccionales nacionales, regionales o distritales a fin de concordar jurisprudencia de su especialidad, a instancia de los órganos de apoyo del Poder Judicial.

En efecto, casi sin darnos cuenta, la constante realización de estos plenos jurisdiccionales en diversos distritos judiciales viene convirtiéndose en una especie de «tradición jurídica». Aunque los plenos jurisdiccionales no son vinculantes, las conclusiones de los mismos son muchas veces aplicadas por los jueces en los casos $\operatorname{concretos}^{4}$ por la fuerza de las razones y los argumentos que en ellos se ofrecen (fuerza persuasiva). Precisamente, el tema abordado en este ensayo está referido a la figura del abandono procesal en el contexto del proceso civil. Muy especialmente, se analiza el problema del abandono de aquellos procesos que contienen pretensiones relacionadas con el derecho de propiedad. El cual ha sido, también, materia de análisis del Pleno Jurisdiccional Nacional Civil y Procesal Civil 2016, realizado en la ciudad de Lima, los días ocho y nueve de julio. En dicho pleno se sostuvo con respecto a estas pretensiones que, al ser consideradas imprescriptibles, no procedería el abandono. Literalmente, se acordó por mayoría lo siguiente: «No se produce el abandono, ya que se trata de pretensiones imprescriptibles vinculadas al derecho de propiedad o a los derechos que se derivan de la misma» (Conclusiones plenarias, 2016).

Por estas razones, el propósito de este breve estudio será, en primer lugar, describir y analizar de modo crítico las dos ponencias expuestas en el referido Pleno Jurisdiccional, así como sus respectivas justificaciones, centrándonos en aquella que finalmente fue asumida en mayoría por

4 En el Perú, los plenos jurisdiccionales son de diversos tipos. Por ejemplo, en relación con la proyección geográfica, pueden ser distritales, regionales y nacionales; atendiendo a los grados de la organización judicial: plenos de jueces de paz letrados, especializados, superiores y supremos; $y$, finalmente, considerando la materia o especialidad, pueden ser plenos de familia, laborales, penales, contencioso-administrativos, constitucionales, civiles y procesal-civiles. Además, se admite combinaciones y variantes de estos criterios, por ejemplo, puede darse el caso que existan plenos jurisdiccional nacional civil y procesal civil. Empero, conviene plantearse la siguiente problemática: si su propósito es propiciar los valores de seguridad y predictibilidad, esta multiplicidad y diversidad no parece ser ideal, más aun si desde 1997 se vienen realizando de manera ininterrumpida todos los años (según información proporcionada por el Poder Judicial del Perú en la página «Plenos J urisdiccionales» al interior de su sitio web). Esta frecuencia puede implicar que para los jueces (pero también para los abogados) sea una tarea realmente titánica y complicada encontrar un criterio entre tantos plenos.

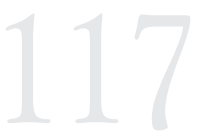

EL PROBLEMA DEL ABANDONO DE LAS PRETENSIONES IMPRESCRIPTIBLES

\section{THE}

ABANDONMENT

ISSUE OFTHE

IMPRESCRIPTIBLE PRETENSIONS 
los jueces superiores con relación al tema en discusión. En segundo lugar, situar el debate en un punto elemental que lamentablemente no fue considerado, pero que, desde nuestra modesta consideración, subyace a lo discutido en el pleno. Dicho punto elemental es la cuestión de si las pretensiones imprescriptibles (como el caso del derecho de la propiedad) realmente merecen tal inmunidad frente al abandono; es decir, cuestionar, desde sus bases, la «necesaria» relación: abandono del proceso-pretensiones imprescriptibles.

\section{SENTIDO Y JUSTIFICACIÓN DEL ABANDONO}

Detengámonos un momento para esclarecer en qué consiste el abandono procesal - también denominado perención o caducidad de instancia (caducidad de instancia es término empleado en el proceso civil español, Ley de Enjuiciamiento Civil 1/2000, libro I, título VI, artículos 236-240) - . Se trata de una institución procesal que se presenta cuando existe una detención o inercia del proceso por un tiempo fijado por ley y que genera inevitablemente la conclusión del proceso, obviamente sin pronunciamiento sobre el fondo (rectius: mérito); razón por la cual algunos la consideran una forma anormal de conclusión del proceso 5 .

Bien entendido, el abandono no puede ser visto como una sanción jurídica, sino, propiamente, como una consecuencia jurídica por la que se concluye el proceso de modo anormal ${ }^{6}$, por encontrarse detenido por un tiempo fijado por ley, con la particularidad de que esta situación no sea causada o provocada por la inacción del juez $z^{7}$. En efecto, se sostiene que existen al menos dos motivos en que se fundamenta. El primero es de carácter subjetivo y ve en la presunta intención de las partes de abandonar el proceso la íntima de la extinción, es decir, se determina por la voluntad de los litigantes. El segundo es de tipo objetivo y se fija en la necesidad de evitar la pendencia indefinida de los procesos, por las consecuencias que esto implica para la seguridad jurídica, en otras

5 Para justificar esta expresión, se ha dicho que la anormalidad de estos especiales fenómenos y de sus consecuentes desenlaces especiales del proceso no significa necesariamente su disconformidad con las normas jurídicas relativas a la terminación del proceso, sino que debe entenderse en el sentido de que la sentencia de fondo dictada a consecuencia de una contradicción, mayor o menor, entre las partes es, de hecho, lo más frecuente y habitual y, de derecho, lo que la ley procesal considera ordinario y común (De la Oliva \& Diez-Picazo, 2000, p. 422).

6 En la comparación jurídica, considerada como una forma de conclusión anormal del proceso, por ejemplo, el Tribunal Supremo español ha establecido que la «caducidad de la instancia es una de las especies del concepto más general de extinción del proceso, entendiendo por extinción toda conclusión anormal producida sin que el proceso haya cumplido su fin, esto es sin que se haya decidido sobre la pretensión en el mismo planteada. Cuando la extinción se causa por inactividad de los sujetos se está ante la caducidad, que se produce, pues, sin acto de clase alguna, por el simple hecho del transcurso del tiempo sin realizar actividad procesal» (STS 17784/1993).

7 En la doctrina italiana, Chiovenda, quien identifica el fenómeno como caducidad, lo concibe de la siguiente manera: «es un modo de extinción de la relación procesal, y que se produce después de cierto período de tiempo, en virtud de la inactividad de los sujetos procesales» (1940, p. 310). También, Carnelutti, explicaba que «El procedimiento se extingue por perención, cuando habiendo asignado un plazo perentorio, por la Ley o por el J uez, para el cumplimiento de un acto necesario a la prosecución, dicho acto no es realizado dentro del plazo» (1959, p. 174). 
palabras, se trata de una cuestión que escapa a la voluntad de las partes de que no se prolongue la duración de los pleitos paralizados (Guasp, 1973, pp. 539-540).

El legislador nacional parece haber entendido bien esta referencia, pues ha regulado el abandono — en el capítulo de las formas especiales de conclusión del proceso del Código Procesal Civil—de la siguiente manera: "Cuando el proceso permanezca en primera instancia durante cuatro meses sin que se realice acto que lo impulse, el juez declarará su abandono de oficio o a solicitud de parte o de tercero legitimado» (CPC, artículo 346). La idea que subyace a esta institución procesal sería que los procesos no pueden durar eternamente sin lograr su propósito natural.

De este modo, el abandono se justifica en la medida en que la misma expresión «proceso» representa per se y en todos los casos un necesario devenir diacrónico de actos dirigidos hacia la consecución de un fin, es decir, una proyección temporal hacia el futuro. Así, toda falta de avance injustificada sería contraria al sentido mismo del proceso ${ }^{8}$. Este sencillo razonamiento calza, precisamente, de modo ideal cuando se habla de proceso (jurisdiccional), pues, desde una perspectiva formal-estructural, implica un conjunto de actos jurídicos (procesales) concatenados armónicamente hacia un propósito: la búsqueda de la verdad, mediante decisiones justas, al menos si nos adherimos a una de las opciones ideológicas típica de los sistemas de Civil Law9. Si esto es así, entonces, es evidente que el hecho de presentarse una detención o inercia que dificulte el avance y desarrollo natural del proceso puede generar un problema en relación con el cumplimiento de su finalidad.

De suceder esto, ciertamente el Estado/juez perdería su legitimidad para continuar su prosecución del proceso, en otras palabras, no existiría justificación de que continúe con el procesamiento de una persona. La pérdida de legitimidad ocurre porque — precisamente-el método que el Estado utiliza para la tutela de las situaciones jurídicas sustantivas, el proceso, ha perdido su propósito por haberse detenido injustificadamente durante un tiempo determinado por la ley.

8 Este sentido es acorde con la propuesta de Guasp, quien afirma que la caducidad de la instancia (para nosotros abandono procesal) «es la extinción del proceso que se produce por su paralización durante cierto tiempo en que no realizan actos procesales las partes» (1973, p. 539).

9 En efecto, por ejemplo, para Taruffo, «La justicia de la decisión no presupone solamente su legalidad, es decir, que se derive de una correcta interpretación y aplicación de las normas, sino también su veracidad, es decir, la determinación de la verdad de los hechos relevantes: la razón fundamental de esto es que ninguna decisión puede considerarse justa si se basa en una determinación falsa 0 errada de los hechos de la causa» (2010, p. 413). Sobre la relación teleológica entre prueba y verdad, véase Ferrer (2007, pp. 29-30).

EL PROBLEMA DEL ABANDONO DE LAS PRETENSIONES IMPRESCRIPTIBLES

THE

ABANDONMENT

ISSUE OFTHE

IMPRESCRIPTIBLE PRETENSIONS 


\section{EL ABANDONO EN UN MUNDO «DE OFICIO»}

Además, otro punto importante que se debe considerar es la relación del abandono con el «principio» ${ }^{10}$ (rectius: regla) de impulso procesal. Antes, sin embargo, conviene recordar que el proceso civil peruano ha sido diseñado y estructurado —en gran parte-bajo la incidencia del principio de oficialidad ${ }^{11}$ (impropiamente denominado «principio» publicístico o inquisitivo), en virtud del cual se le atribuye al juez ciertas potestades, técnicas e iniciativas para el desarrollo y conducción del proceso hacia el cumplimiento de su propósito. Precisamente, una de las manifestaciones de tal principio es el llamado «principio» (rectius: regla) de impulso de oficio, en virtud del cual la continuación o avance del proceso se encuentra bajo responsabilidad del juez ${ }^{12}$. El cual normativamente es descrito en estos términos: «El juez debe impulsar el proceso por sí mismo, siendo responsable de cualquier demora ocasionada por su negligencia. Están exceptuados de este impulso los casos expresamente señalados en este Código», (CPC, título preliminar, art. 2, § 2). En otras palabras, en el proceso civil peruano $-\mathrm{y}$ en los procesos no penales en general — la regla es que el proceso se impulsa no a pedido de parte, sino propiamente de oficio, esto es, por el órgano jurisdiccional. Por tanto, las partes en el proceso, prima facie, no tendrían el deber —al menos no formalmente - de estar presentando escritos para propiciar su continuación al estado correspondiente, sino que esta tarea corresponde al mismo jue $z^{13}$.

Por tanto, la pregunta sería cómo se justifica la figura del abandono en un proceso impulsado por el juez, dicho en términos alegóricos, «en un mundo de oficio»; pues se supone que el juez es el llamado a velar por la continuación y el impulso del proceso. Si el impulso del proceso se le hubiese encargado a las partes (impulso de parte), la razón sería más que evidente: la falta de interés procesal (estado de necesidad de

10 Sobre el particular, el profesor De la Oliva es enfático en sostener que «desde varios puntos de vista, es erróneo y perturbador denominar "principios" a todos los criterios generales en virtud de los cuales se opta por regular de un modo o de otro el proceso o ciertos aspectos 0 actuaciones de este. Para la mayoría de esos criterios resulta preferible utilizar los conceptos y términos de "reglas" o "máximas". Como he repetido en muchas ocasiones, cuando todo son principios, nada es principio. Y lo mismo sucede - y con impaciencia lo repito también una y otra vez- cuando a cualquier posibilidad de actuación humana se le denomina "derecho": cuando todos son "derechos", nada es derecho. Semejante amplitud conceptual no conduce a nada positivo» (2012, p. 245).

$11 \mathrm{EI}$ profesor De la Oliva explica que el principio de oficialidad es aquel «derivado del intenso interés público predominante en ciertas materias, por el cual el proceso, su objeto, los actos procesales y la sentencia no están subordinados a la disposición de los sujetos jurídicos particulares en relación con la tutela de los derechos e intereses legítimos, sino que dependen de que aquel interés se ponga de manifiesto al tribunal y se haga valer por otros órganos públicos ante situaciones subsumibles en supuestos taxativamente determinados por la ley» (de la Oliva, Diez-Picazo, Giménez \& Vegas, 2012, p. 208).

12 Con relación al impulso procesal, en general, Eduardo Couture explica que «Se denomina impulso procesal al fenómeno cuya virtud asegura la continuidad de los actos procesales y su dirección hacia el fallo definitivo» (2002, p. 142).

13 Este razonamiento se ve plasmado en la siguiente posición de la Corte Suprema: «si bien la figura del abandono importa la inactividad procesal, su declaración no puede obviar la obligación del juzgador en su condición de director del proceso, de impulsarlo aún sin necesidad de que la otra parte lo solicite y cuando el estado mismo corresponda» (Casación 2422-2013 J UNíN, fundamento 8). 
tutela jurisdiccional); pero icómo se puede concebir que un proceso se detenga, cuando su continuación es labor del juez?

La razón es que pueden existir algunos casos en que la inercia del proceso, pese al impulso de oficio, no se deba a la desidia o negligencia del juez por continuar el trámite o al estar pendiente la emisión de una resolución (CPC, artículo 350, 5), sino propiamente a la responsabilidad de las partes. Esto sucede, por ejemplo, cuando se requiere el cumplimiento - a cargo de las partes - del pago de arancel para una determinada actuación judicial, sin el cual no podría realizarse o, en general, en cualquier caso en que se exija del demandante o demandado determinada prestación, pero estos simplemente dejan pasar el tiempo sin realizarla ${ }^{14}$. Al menos eso se desprende del artículo 350, 4 del CPC: «salvo que estuviera pendiente actuación cuya realización dependiera de una de las partes».

Es en estas circunstancias donde se produciría el abandono, como una consecuencia jurídica por la falta manifiesta de interés de la parte (demandante) ante el incumplimiento de lo requerido, dentro de un plazo previsto en la ley ${ }^{15}$. Situación que, a la verdad, se debe presentar de manera excepcional, pues siempre el juez, conforme al impulso de oficio, debe ser el primer comprometido en tomar todas las medidas y emitir todas las resoluciones para el necesario avance del proceso ${ }^{16}$.

\section{IV.EL ABANDONO ANALIZADO EN EL PLENO JURISDICCIONAL NACIONAL CIVIL}

Precisamente, en el último Pleno Jurisdiccional Nacional Civil y Procesal Civil, realizado en la ciudad de Lima (para una revisión de todos los temas de debate, ponencias, justificaciones, materiales bibliográficos y jurisprudenciales utilizados en el citado pleno jurisdiccional, véase
EL PROBLEMA DEL ABANDONO DE LAS PRETENSIONES IMPRESCRIPTIBLES

\section{THE}

ABANDONMENT

ISSUE OF THE

IMPRESCRIPTIBLE PRETENSIONS

14 Esta idea parece ser acorde al sentido expresado por la Corte Suprema, para quien el abandono procesal es «un medio procesal a través del cual se extingue un proceso por falta de actividad idónea de los sujetos procesales» (Casación 884-2003 LAMBAYEQUE, citada en Casación 48052010 LIMA).

15 Sobre el particular, la Corte Suprema ha expresado que «el abandono implica dos factores combinados: el tiempo y la inactividad procesal; que provoca la culminación de la instancia y, por ende, del proceso sin declaración sobre el fondo en razón de la inactividad procesal de las partes. Lo que realmente sanciona el abandono es la negligencia manifiesta del litigante, que con su inactividad deja paralizado el proceso. Dicha inactividad tiene que ser medida a través de determinados plazos que la norma regula en cuatro meses» (Casación 4805-2010 LIMA).

16 Por ello llama la atención el hecho de que el abandono se declare también de oficio (CPC, artículo 346). Se trata de una particular disposición normativa, cuyo cumplimiento ha sido "recordado» a todos los jueces por el mismo Consejo Ejecutivo del Poder Judicial, mediante la resolución administrativa 373-2014-CE-PJ, en la que también se requiere - a la Gerencia General del Poder J udicial- incorporar en el Sistema Integrado J udicial (SIJ) un medio para que alerte al juez cuando debe declarar el abandono. Empero, la experiencia y la historia han demostrado que las medidas «urgentes» para solucionar problemas urgentes no siempre son las más apropiadas o idóneas, más aun si se llevan a cabo de forma acrítica, sin analizar detenidamente el sentido y fundamento de cualquier institución. Adicionalmente, habría que llamar la atención sobre lo sorpresiva que resulta la intervención del Consejo Ejecutivo en aspectos que son estrictamente jurisdiccionales. 
Centro de Investigaciones Judiciales, 2016), el primer tema que se debatió estaba relacionado con lo explicado anteriormente, es decir, con el abandono y su improcedencia en los procesos en donde se analice pretensiones imprescriptibles, específicamente, respecto de aquellas vinculadas con el derecho de propiedad. La cuestión fue la siguiente: ise produce el abandono en los procesos en los que se discuten pretensiones vinculadas al derecho de propiedad y a los derechos que se derivan de este? Para dar respuesta a tal problema, la organización del pleno estableció previamente dos ponencias entre las cuales los participantes, luego del debate respectivo, debieron elegir y votar para llegar a un acuerdo.

Analicemos, en primer lugar, cada una de estas posiciones planteadas. Veamos, la primera de ellas era en favor de la procedencia del abandono sobre los procesos en donde se tutele el derecho de propiedad, descrita bajo la siguiente fórmula: «Sí se produce el abandono debido a que se trata de pretensiones que no tienen establecidas las condiciones de imprescriptibles por la ley» (Centro de Investigaciones Judiciales, 2016, p. 15). Se trata de una posición que parte de la siguiente premisa: para que una pretensión tenga la calidad de imprescriptible, debe ser manifiesta en el ordenamiento legal, es decir, es la ley quien fija dicho carácter. A contrario sensu, sino hay regla expresa, simplemente sí les afectaría la prescripción, como pasa en la mayoría de casos, serían prescriptibles ${ }^{17}$. Dicho de otra manera, desde esta visión, la regla general sería la siguiente: se presume que la prescripción es aplicable a todas las pretensiones y para que esto no suceda, debe darse una regla expresa.

En concreto, respecto de las pretensiones relativas al derecho de propiedad, no existe disposición normativa expresa en nuestro ordenamiento que le reconozca tal condición, por lo que - siempre desde esta postura - resultaría correcto que proceda el abandono, dado que solo las pretensiones que son imprescriptibles están exentas de tal consecuencia. Esto es así conforme al artículo 350, 3 del CPC, que establece que no hay abandono «En los procesos en que se contiendan pretensiones imprescriptibles». Básicamente, este fue el razonamiento — palabras más, palabras menos_ que se expuso como justificación de tal posición en el pleno jurisdiccional.

No obstante, a nuestro modo de ver, se trata de una posición - por decir lo menos-excesivamente devota de la ley (o disposición normativa) en la que subyace una preferencia u opción ideológica del derecho desde una óptica muy particular, diría un enfoque positivista llevado a sus

$17 \mathrm{Si}$ revisamos con detenimiento el Código Civil peruano (donde se encuentran las reglas de prescripción) advertiremos que hay derechos que son calificados de modo expreso y literal con la calidad de imprescriptibles, como el caso del derecho de la propiedad de las tierras de las comunidades campesinas y nativas (artículo 136), petición de herencia (artículos 664 y 865), reivindicación de un bien salvo la prescripción adquisitiva (artículo 927), entre otros. 
últimos términos. Esto, desde mi modesta perspectiva, no hace otra cosa que alentar ese clásico apego o predilección por «respetar» a toda costa el «texto expreso de la ley», pese a que pueden existir razones fuertes que acaben por desvirtuarla o superarla.

La segunda ponencia rechaza la idea de que proceda el abandono en estos casos, ya que las pretensiones relativas al derecho de propiedad sí tienen el carácter de imprescriptibles. Para ser exactos, se dijo lo siguiente: «No se produce el abandono, ya que se trata de pretensiones imprescriptibles vinculadas al derecho de propiedad o a los derechos que se derivan de la misma» (Centro de Investigaciones Judiciales, 2016, p. 15). Para justificar tal posición, se utilizaron expresiones como «esencia» o «naturaleza», dirigidas a justificar que el derecho no solo se limita a meras prescripciones legales, sino que en él confluyen diversos elementos que, desde luego, determinan que el caso del derecho de propiedad sea imprescriptible — como se sabe, desde mucho hace tiempo existe una clara tendencia (con algunas excepciones) a considerar que tanto los derechos de propiedad como los catalogados como personalísimos son imprescriptibles- Sin embargo, existe una omisión en exponer puntualmente las razones y argumentos por las que el derecho de propiedad, desde su posición, tendría el carácter de imprescriptible y no darlo por sentado (indistintamente de que haya o no previsión legal).

Luego de la respectiva ponencia del teórico — magistrado invitadoy del sucesivo debate de los jueces superiores civiles (Poder Judicial del Perú, 2016a, 2016b), finalmente, en el segundo día del pleno jurisdiccional, los jueces superiores asistentes votaron en mayoría por la primera postura. Es decir, se decidió que no procede el abandono procesal, dado que el derecho de propiedad (y los derechos derivados de él) tiene la calidad de imprescriptible, el cual es un supuesto excepcional en el que no procede el abandono.

\section{ELPROBLEMADELAS PRETENSIONESIMPRESCRIPTIBLES}

Como hemos podido apreciar, en el tema analizado, el punto de debate se centró únicamente en esclarecer si el derecho de propiedad tendría la calidad de imprescriptible, para establecer si procede $-\mathrm{O}$ no- el abandono. Si bien este tema ciertamente necesitaba ser esclarecido, consideramos que debió plantearse anteriormente la necesidad de determinar si realmente las pretensiones imprescriptibles no deben estar expuestas al abandono. Dicho de otra manera, ipor qué las pretensiones imprescriptibles — como el derecho de propiedad-gozan de tal protección? (para un breve comentario sobre este problema, véase Alfaro, 2015; ensayo que fue considerado en el material de trabajo del referido pleno jurisdiccional. Sin embargo, el problema ya había sido

EL PROBLEMA DEL ABANDONO DE LAS PRETENSIONES IMPRESCRIPTIBLES

THE

ABANDONMENT

ISSUE OFTHE

IMPRESCRIPTIBLE

PRETENSIONS 
advertido algunos años antes, Alfaro, 2010, p. 189). ¿Cuáles son las razones que justifican tal inmunidad? ¿Qué tan fuertes o sólidas son esas razones como para seguir considerándolas?

Analicemos con detenimiento las razones en que se sustenta tal protección. Recordemos que la característica de que una pretensión sea imprescriptible significa que no se extiende sobre ella la prescripción. Dicho en otros términos, bajo tal cualidad, el demandante tendría la posibilidad de iniciar un proceso de manera sempiterna sin que el tiempo extinga tal posibilidad, por tanto, pueden interponerse simplemente cuando las personas lo consideren conveniente. En este escenario, las preguntas que se imponen serían las siguientes: ¿Qué relación existe entre una pretensión imprescriptible y el hecho de que no pueda caer en abandono? ¿Qué virtud tienen esas pretensiones que las hacen inmunes al abandono? Francamente, no se encuentra vinculación alguna, ya que se trata de situaciones jurídicas distintas. La primera es una cualidad de algunas situaciones jurídicas sustantivas y la segunda es una situación puramente procesal por la inercia del proceso ${ }^{18}$. El hecho de que pueda iniciarse un proceso con tales pretensiones en cualquier momento, sin miedo a que ya no puedan ejercerse por vencimiento de un plazo fijado por ley, no se relaciona de modo alguno con el abandono. Fácilmente puede darse el caso de que la inercia de estos procesos pueda ser —naturalmente - imputable también a las partes. ¿Acaso no sería suficiente privilegio el hecho de que el demandante pueda demandar en cualquier momento, sin riesgo de que prescriba su derecho (antes del proceso), como para que además ahora se lo blinde con la inmunidad procesal (durante el proceso) de no incurrir en abandono?

En caso de que estas razones no fuesen suficientes, veamos si nuestra disposición normativa, la cual prohíbe la declaración de abandono procesal frente a pretensiones imprescriptibles (CPC, artículo 350, 3), tiene algún parangón o punto de referencia contemporánea en algunos sistemas procesales del Civil Law. Es decir, examinemos si existen ordenamientos jurídicos en donde se establece tal proscripción legal. Así, por ejemplo, en el sistema procesal civil español, en concreto, en la Ley de Enjuiciamiento Civil, se puede observar que en toda la dispersión normativa (artículos 236-240) relativa a la llamada caducidad de instancia (para nosotros abandono), los únicos supuestos de exclusión — esto es, sobre los que no podría aplicarse la caducidad - serían cuando el procedimiento hubiere quedado paralizado por fuerza mayor o por cualquier otra causa contraria o no imputable a la voluntad de las

18 En esta misma línea de reflexión, Ariano, respecto de las pretensiones vinculadas con el derecho de propiedad, se hace las siguientes preguntas: «¿Por qué un proceso de partición no puede morir por abandono y sí, en cambio, uno cuyo objeto sea una simple condena al pago de una suma de dinero? Si de lo que se trata es que los procesos no sean eternos, molestando más de lo tolerable al órgano judicial, ¿ por qué uno, frente a la inercia de las parte, podría eternizarse y el otro no? Misterio» (2004, p. 50). 
partes o interesados (artículo 238) y en el caso de las actuaciones para la ejecución forzosa (artículo 239). Sin embargo, no existe ningún caso que prohíba su aplicación ateniendo a la naturaleza de las pretensiones (prescriptibles o imprescriptibles), lo que significa que necesariamente procede en todos los procesos, indistintamente del tipo de pretensiones que se formule.

Situación similar acontece en el proceso civil francés, pues en el código procesal civil francés no se aprecia alguna regla semejante a la analizada en sus disposiciones normativas vinculadas con la péremption d'instance (caducidad de instancia) (Code de procédure civile, artículos 386-393). Por lo tanto, en el sistema francés, el hecho de que en un proceso se analice pretensiones imprescriptibles y el proceso eventualmente haya superado los dos años ${ }^{19}$ sin que se realicen actuaciones no sería óbice a que se declare la caducidad de instancia.

Así, queda completamente demostrado que no existen razones fuertes y menos de carácter comparativo que justifiquen la exención al abandono de los procesos en los que se resuelvan pretensiones que tienen la cualidad de imprescriptibles como, por ejemplo, las relativas al derecho de propiedad. Por el contrario, existe más de una razón para pensar que deberían ser, al igual que toda pretensión procesal, expuestas al abandono. Hubiese sido preferible que el pleno jurisdiccional abordara este específico problema y viese las ponencias que lo sustenten, pues — en realidad — de haberse superado dicho problema, el tema elegido resultaría simplemente superfluo.

\section{REFLEXIONES FINALES}

En este breve ensayo hemos intentado, sobre todo, desvirtuar el nexo entre abandono procesal y pretensiones imprescriptibles (CPC, artículo $350,3)$ proporcionando algunos argumentos para postular que no existen razones jurídicas que justifiquen tal prescripción legal. Así, según nuestro modesto parecer, se debe considerar (de lege ferenda) de manera urgente su inmediata modificación legal. Se debe eliminar tal protección jurídica, máxime - para reforzar esta propuesta- si, revisando el derecho comparado, se advierte que esta disposición normativa no tiene parangón alguno en otros sistemas procesales —al menos entre los más prestigiosos - como el ordenamiento español o francés.

El Poder Judicial hizo bien en elegir al instituto del abandono procesal como primer tema analizado en el Pleno Jurisdiccional Nacional Civil y

19 A diferencia del proceso civil peruano, en el modelo francés, el plazo legal de inactividad procesal del proceso es de dos años, conforme a lo previsto en el artículo 386 del Codede procédure civile, en donde se establece lo siguiente: «Se producirá la caducidad de la instancia cuando ninguna de las partes realizara actuaciones en el proceso durante dos años».

EL PROBLEMA DEL ABANDONO DE LAS PRETENSIONES IMPRESCRIPTIBLES THE ABANDONMENT ISSUE OFTHE IMPRESCRIPTIBLE PRETENSIONS 
Procesal Civil, dado que los juzgados, en general, manejan una elevada carga procesal y dicho instituto, ciertamente, coadyuva a disminuir tal situación crítica. Empero, la cuestión específica planteada (procedencia en las pretensiones imprescriptibles vinculadas al derecho de propiedad) no fue del todo acertada. Se perdió una gran oportunidad para discutir el problema medular que es anterior o previo a la cuestión analizada.

A decir verdad, existe casi un consenso generalizado, en la doctrina y jurisprudencia, en que las pretensiones relativas al derecho de propiedad tienen el carácter de imprescriptible, pese a que esto no esté expresamente previsto en el Código Civil, por lo que el «esclarecimiento» de esta específica cuestión no era del todo necesario. El verdadero problema relativo al abandono procesal, el que realmente debió ser analizado en dicho pleno jurisdiccional, es esclarecer cuál es la relación que existe entre el abandono procesal y las pretensiones imprescriptibles, es decir, saber si, en efecto, las pretensiones que tienen tal calidad deben o no ser expuestas al abandono y ofrecer razones que justifiquen una u otra posición.

\section{REFERENCIAS}

Alfaro, L. (2010). El periodo de tiempo de la suspensión legal de un proceso no se computa para el abandono [opinión]. Diálogo con la Jurisprudencia, 15 (136), 189.

Alfaro, L. (2015). Impulso de oficio vs. abandono del proceso [comentario]. Diálogo con la Jurisprudencia, 20(196), 149.

Ariano, E. (2004). Conclusión del proceso por inactividad de las partes y prescripción. Diálogo con la jurisprudencia, 9(64), 43-52.

Carnelutti, F. (1959). Instituciones del proceso civil (t. 2). Buenos Aires: Ediciones Jurídicas Europa-América.

Centro de Investigaciones Judiciales (2008). Guía metodológica de Plenos Jurisdiccionales aprobada por el consejo ejecutivo del Poder Judicial. Recuperado de https:/www.pj.gob.pe/wps/wcm/connect/7407c18043eb77c79256d34684c62 36a/CS_D_CIJ_guia_plenos.pdf?MOD=AJPERES

Centro de Investigaciones Judiciales (2016). Pleno Jurisdiccional Nacional Civil y Procesal Civil. Recuperado de https://www.pj.gob.pe/wps/wcm/connect/7fe7ef 004de035ac9b7cdb73e0b6364e/PLENO+JURISDICCIONAL+NACIONAL $+\mathrm{CIVIL}+\mathrm{Y}+\mathrm{PROCESAL}+$ +(2).pdf?MOD $=$ AJPERES

Chiovenda, G. (1940). Instituciones de derecho procesal civil (t. 3). Madrid: Revista de Derecho Privado.

Conclusiones plenarias (2016, 08 y 09 de julio). Pleno Jurisdiccional Nacional Civil y Procesal Civil, Lima. Recuperado de https:/www.pj.gob.pe/wps/wcm/connect/ d88b1f804dd73aOeacaOfe 73e0b6364e/Nacional + civil+y+ procesal+ civil.pdf? $\mathrm{MOD}=\mathrm{AJPERES} \& C A C H E I D=\mathrm{d} 88 \mathrm{~b} 1 \mathrm{f} 804 \mathrm{dd} 73 \mathrm{a}$ 0eacaOfe 73e0b6364e 
Couture, E. (2002). Fundamentos del derecho procesal civil (4ta ed.). Buenos Aires: $\mathrm{B}$ de $\mathrm{F}$.

De la Oliva, A. \& Diez-Picazo, I. (2000). Derecho procesal civil: El proceso de declaración. Madrid: Centro de Estudios Ramón Areces (CERA).

De la Oliva, A. (2012). Prudencia versus ideología: De nuevo sobre el papel del juez en el proceso civil. Ius et Praxis, 18(2), 243-294. doi: 10.4067/S071800122012000200009

De la Oliva, A., Diez-Picazo, I. \& Vegas, J. (2012).Los principios del proceso. En Curso de derecho procesal civil I: Parte general (lección 10). Madrid: Centro de Estudios Ramón Areces (CERA).

Ferrer, J. (2007). La valoración racional de la prueba. Madrid: Marcial Pons.

Guasp, J. (1973). Derecho procesal civil (t. 1, 3ra ed.). Madrid: Instituto de Estudios Políticos.

Manjón-Cabeza, A. (2008). ¿Son vinculantes los Acuerdos del Pleno no Jurisdiccional de la sala segunda del TS? (A propósito del Acuerdo de 18 de julio de 2006). Revista Electrónica de Ciencia Penal y Criminología, 10,1-25. Recuperado de http://criminet.ugr.es/recpc/10/recpc10-02.pdf

Poder Judicial del Perú (s. f.). Plenos Jurisdiccionales [página web]. Recuperado de https:/www.pj.gob.pe/wps/wcm/connect/cij/s_corte_suprema_utilitarios/as home/as_cij/as_plenos_jurisdiccionales/

Poder Judicial del Perú (2016a, 14 de julio). 08/07/2016 - Pleno Jurisdiccional Nacional Civil y Procesal Civil (Día 1) [video]. Recuperado de https://www. youtube.com/watch? $\mathrm{v}=\mathrm{S} 4 \mathrm{dGjN} 4 \mathrm{tjBg}$.

Poder Judicial del Perú (2016b, 12 de julio). 09/07/2016 - Pleno Jurisdiccional Nacional Civil y Procesal Civil (Día 2). Recuperado dehttps://www.youtube.com/ watch? $=\mathrm{J} 4 \mathrm{Mgu} 8 \mathrm{U} 4 \mathrm{La} 8$

Sacco, R. (1992). Introduzione al diritto comparato.Turín: Utet.

Somma, A. (2015). Introducción al derecho comparado. Madrid: Universidad Carlos III de Madrid.

Taruffo, M. (2007). Precedente y jurisprudencia. Precedente. Revista Jurídica, 8699. doi: 10.18046/prec.v0.1434

Taruffo, M. (2010). Páginas sobre justicia civil. Madrid: Marcial Pons.

Taruffo, M. (2012). El precedente judicial en los sistemas de Civil Law. Ius Et Veritas, 45, 88-95. Recuperado de http://revistas.pucp.edu.pe/index.php/ iusetveritas/article/view/11991/12559

Jurisprudencia, normativa y otros documentos legales Casación 2422-2013 JUNÍN (Corte Suprema de Justicia de la República [Perú], Sala Civil Transitoria, 13 de agosto de 2014).

EL PROBLEMA DEL ABANDONO DE LAS PRETENSIONES IMPRESCRIPTIBLES

THE

ABANDONMENT ISSUE OF THE IMPRESCRIPTIBLE PRETENSIONS 
Casación 4805-2010 LIMA, (Corte Suprema de Justicia de la República [Perú], Sala Civil Permanente, 13 de octubre de 2011).

Code de Procédure Civile [Código Procesal Civil, Francia].Traducción de Fernando Gascón Inchausti. Recuperado de https://www.legifrance.gouv.fr/ Traductions/Catalogue-des-traductions

Código Civil [Perú].

Código Procesal Civil [CPC] [Perú].

Ley de Enjuiciamiento Civil, ley 1/2000, BOE-A-2000-323, Boletín Oficial del Estado [BOE] [España], 7 (08 de enero de 2000, pp. 575-728).

Ley Orgánica del Poder Judicial, Presidencia de la República [Perú], decreto supremo 017-93-JUS, Diario Oficial El Peruano (02 de junio de 1993). Tercera edición oficial del Texto Único Ordenado (2012), recuperado de https://www. minjus.gob.pe/wp-content/uploads/2014/09/DGDOJ-Ley-Orgánica-PoderJudicial-y-Ministerio-Público.pdf

Resolución administrativa 373-2014-CE-PJ, Diario Oficial El Peruano (30 de diciembre de 2014).

STS 17784/1993, ECLI: ES:TS:1993:17784, (Tribunal Supremo [España], 29 de junio de 1993). 